Jul 1st, 12:00 AM

\title{
On Line Simulation System for Industrial Accidents
}

\author{
R. Bellasio \\ R. Bianconi
}

Follow this and additional works at: https://scholarsarchive.byu.edu/iemssconference

Bellasio, R. and Bianconi, R., "On Line Simulation System for Industrial Accidents" (2002). International Congress on Environmental Modelling and Software. 153.

https://scholarsarchive.byu.edu/iemssconference/2002/all/153

This Event is brought to you for free and open access by the Civil and Environmental Engineering at BYU ScholarsArchive. It has been accepted for inclusion in International Congress on Environmental Modelling and Software by an authorized administrator of BYU ScholarsArchive. For more information, please contact scholarsarchive@byu.edu, ellen_amatangelo@byu.edu. 


\title{
On Line Simulation System for Industrial Accidents
}

\author{
$\underline{\text { R. Bellasio and R. Bianconi }}$ \\ ENVIROWARE srl, Centro Direzionale Colleoni, 20041 Agrate Brianza (Mi). Italy \\ (rbellasio@enviroware.com)
}

\begin{abstract}
Mathematical models are important tools to evaluate the consequences of possible industrial accidents. These models are often difficult to apply because they require trained users, and graphical user interfaces can be of great help. An on line simulation system has been developed and is installed as a serverside application on the internet at www.enviroware.it. The system currently contains two models, EXPSYS is suitable to carry out consequence analysis and allows the user to evaluate the area impacted by industrial accidents such as explosions, fires and releases of toxic or flammable gases. TOXFLAM is an analytic model suitable for simulating the atmospheric dispersion of gases lighter than air. Other simulation models will be added to the system in the future. The modelling system allows each user to upload her/his own topography files and to georeferentiate them. The topography files are then used both during the input phases, for example for positioning the source by means of the mouse pointer, and during the output phase for plotting the concentration fields or the area at risk. Each simulation, composed of input and output data, can be saved in the personal folder of the user. The simulation data can then be reloaded to view the results, can be used to start new simulations and can be removed when they are no longer useful. A simulation system based on the internet has some advantages with respect to single simulation models used off-line. For example all the users share a unique simulation tool, they can suggest modifications and improvements are readily available to all. The graphical user interface created with HTML makes the models more friendly and easy to use, and available on any platform, independently from the operating system. Moreover output data can be available, as usual, both as ASCII data and directly processed to give a graphical result.
\end{abstract}

Keywords: Industrial accidents; On line simulation, Atmospheric dispersion

\section{INTRODUCTION}

Many of the substances used by industries are potentially dangerous if accidentally released into the atmosphere since they can be either toxic or flammable, or both. It is of fundamental importance to estimate the damages due to possible accidental releases of such substances, because this process allows one, for example, to set up emergency plans, to evaluate the possibilities of a domino effect and to plan a correct land use. A reliable estimation of the damages of an accident allows one to limit the accident consequences (i.e. to increase "protection").

At the European level a Directive $(96 / 82 / \mathrm{EC}$ or Seveso II) is devoted to prevention of majoraccidents and limitation of their consequences. It applies to establishments where dangerous substances are present in quantities equal to or in excess of specific thresholds.

The term major accident stands for "an occurrence such as a major emission, fire, or explosion resulting from uncontrolled developments in the course of the operation of any establishment covered by this Directive, and leading to serious danger to human health and/or the environment, immediate or delayed, inside or outside the establishment, and involving one or more dangerous substances".

The Directive contains obligations to set up internal and external emergency plans, and to evaluate the possibility of domino effects.

Mathematical models are very useful tools to simulate the consequences of an accident and, therefore, they give a lot of indications on how to decrease such consequences. Often models for the simulation of industrial accidents are demanding due to the complexity of the accident evolution (simulation of the source term, ignition, non ignition, delayed ignition, dense vapour, buoyant vapour, ...), to the complexity of the input data required and, sometimes, to the absence of graphical user interfaces helping the users to insert the input data and to interpret the output.

This paper describes a simulation system available on the internet that helps to estimate the 
consequences of possible accidents in a fast and reliable way. The system can be used everywhere, provided a personal computer with internet connection is available.

\section{MODELS THEORY}

The system currently contains two models: EXPSYS is suitable to carry out consequence analysis and allows one to evaluate the area impacted by industrial accidents such as explosions, fires and releases of toxic or flammable gases; TOXFLAM is an analytic model suitable for simulating the atmospheric dispersion of light vapours.

\subsection{EXPSYS}

EXPSYS simulates releases of liquids or vapours that can be toxic and/or flammable. It is based on the algorithms described by the US-EPA [1999] to obtain results in a short time. This model determines the area where the release has its effects. In particular:

- the region where the concentration of a toxic vapour is above a specific toxicity level,

- the region where the concentration of a flammable vapour is above the lower limit of flammability,

- the region where a pool fire (generated by the ignition of a liquid on the ground) damages a user specified target,

- the region where a fireball (generated by a mass of fuel burning and rising into the sky) damages a user specified target,

- the region where a UVCE (Unconfined Vapour Cloud Explosion) damages a user specified target.

Starting from the storage conditions the model determines the release rate and duration. If the substance is released in liquid phase and if the atmospheric dispersion of the vapour is required, the evaporation rate is also calculated. The equations of the model have been recently described in Quaranta et al. [2002], and will not be reported here.

The release rate in liquid phase is described by means of the well known Bernoulli equation. It depends from variables such as hole area, liquid height above the hole, liquid density, storage pressure and atmospheric pressure. Once a liquid pool is formed on the ground, its evaporation rate is calculated using the equation described in US-EPA [1999]. The evaporation rate depends on different variables, among which is the pool area, that is calculated assuming that the pool spreads until its height is equal to $1 \mathrm{~cm}$. If a containment dike is present the pool area is calculated in a different way: if the liquid volume divided by the dike area exceeds the dike height, part of the liquid will exit from the dike and will spread until its height equals $1 \mathrm{~cm}$. The pool area is the sum between the dike area and the area of the liquid outside the dike.

If the substance is released directly as a vapour, the release rate is described as illustrated in Woodward and Mudan [1991].

The vapour release rate, or the evaporation rate from a liquid pool, and the respective duration, are used for calculating the atmospheric dispersion of the vapour. Pre-calculated tables are used for giving the distance of the end point of concern as function of two sets of meteorological conditions: a very stringent one characterised by a wind speed of $1.5 \mathrm{~m} \mathrm{~s}^{-1}$ and $\mathrm{F}$ Pasquill Gifford atmospheric stability class, and a more probable one characterised by a wind speed of $3.0 \mathrm{~m} \mathrm{~s}^{-1}$ and D stability class. Other variables affecting the results of the pre-calculated tables are the vapour relative density (with respect to the air), the release or evaporation rate, the release or evaporation duration, the ground type over which dispersion occurs (rural or urban), and the level of concern (a toxicity level or the lower flammability level). The tables give values representing the maximum distance at which the vapour concentration falls below the toxicity level or the lower flammability level. The minimum end point distance reported by the tables is $160 \mathrm{~m}$, while the maximum one exceeds $40 \mathrm{~km}$.

The model calculates the effects of unconfined vapour cloud explosions (UVCEs) using the TNT equivalence method [Marshall, 1987]. The TNT equivalent mass is calculated starting from the mass of flammable vapour between LFL and UFL and from the heat of combustion of the vapour. Many hydrocarbons have an heat of combustion about 10 times greater than TNT. Other coefficients are considered for calculating the TNT equivalent mass; they account for the energy fraction of the cloud that participates to the explosion, and for the difference between the unconfined vapour explosion and the TNT confined one. From the TNT equivalent mass the overpressure at specified distances is obtained. Since relations between overpressure values and damages to specific "targets" are known, the user selects the target of interest (e.g. human beings, buildings, walls, ...) and the model determines the region where the target is affected by the explosion.

The model simulates the thermal radiation due to a pool fire or a fireball assuming a point flame. This 
assumption is acceptable when the target is far from the fire (about 5 times the fire radius). Moreover, for the pool fire, other features are not calculated, as for example flame tilting or flame drag. The thermal radiation per unit area received by a target at a specific distance from a pool fire is calculated as indicated by US-EPA [1999] as function of combustion rate, atmospheric trasmissivity and distance itself. The combustion rate is calculated as a function of heat of combustion, heat of vaporisation, specific heat, boiling temperature and atmospheric temperature. A similar equation, giving the thermal radiation for unit area over a target at a given distance, holds for the fireball.

\subsection{TOXFLAM}

TOXFLAM is an evolution of MRBT [Bianconi and Tamponi, 1993], an analytical model describing the atmospheric dispersion of inert or linearly-decaying substances with air density, released at variable rate. TOXFLAM is based on a general solution of the diffusion equation that describes instantaneous, finite or continuous releases. The model accounts for the finite height of the planetary boundary layer and uses a mean wind speed obtained from the integration of the vertical wind profile. The non-stationary mathematical solution implemented makes TOXFLAM more general than other regulatory models based on the Gaussian solution. The model computes the concentration field at any time, the maximum reachable concentration at any location, the average concentration over a finite time period (dose) and the infinite exposure. In the frame of the simulation system the computations have been limited to the concentration field.

The model solves analytically the following non stationary atmospheric dispersion equation:

$\frac{\partial C}{\partial t}=K_{x} \frac{\partial^{2} C}{\partial x^{2}}+K_{y} \frac{\partial^{2} C}{\partial y^{2}}+K_{z} \frac{\partial^{2} C}{\partial z^{2}}-u \frac{\partial C}{\partial x}-\lambda C$

$+q\left[\eta(t)-\eta\left(t-t_{r}\right)\right] \delta(x) \delta(y) \delta(z-h)$

The initial condition $(\mathrm{t}=0)$ is null concentration at any point $(\mathrm{x}, \mathrm{y}, \mathrm{z})$, while the boundary conditions are null concentration for each time and $\mathrm{z}$ coordinate until the PBL height $\mathrm{H}$ when $\mathrm{x}$ and $\mathrm{y}$ tend to infinity, and vertical derivative of concentration equal to zero at PBL height $\mathrm{H}$ when time, $\mathrm{x}$ and $\mathrm{y}$ coordinates tend to infinity. In the above equation $\mathrm{C}$ is concentration, $\mathrm{h}$ is the actual release height, $\mathrm{x}, \mathrm{y}$ and $\mathrm{z}$ are the coordinates of the receptor points, $\mathrm{u}$ is the wind component along the $\mathrm{X}$ axis, $\lambda$ is the decay constant, $\mathrm{K}_{\mathrm{x}}, \mathrm{K}_{\mathrm{y}}$ and $\mathrm{K}_{\mathrm{z}}$ are diffusion coefficients along the three axis, $\delta$ is the
Dirac function, $\eta$ is the Heaviside function, $t_{r}$ is the release duration and $\mathrm{q}$ is the emission rate.

The solution of equation (1), that allows to describe the temporal evolution of the concentration field,.is [Bianconi and Tamponi, 1993] when $0<\mathrm{t}<\mathrm{t}_{\mathrm{r}}$ :

$$
\begin{aligned}
C(t, x, y, z)= & \frac{G}{2}\left\{\operatorname{erf}\left(\frac{1}{\sigma_{x} \sqrt{2}}\left(x-\frac{\lambda \sigma_{x}^{2}}{u}\right)\right)-\right. \\
& \left.\operatorname{erf}\left(\frac{1}{\sigma_{x} \sqrt{2}}\left(x-\frac{\lambda \sigma_{x}^{2}}{u}-u t\right)\right)\right\}
\end{aligned}
$$

while when $t \geq t_{r}$ the solution is:

$$
\begin{aligned}
C(t, x, y, z)= & \frac{G}{2}\left\{\operatorname{erf}\left(\frac{1}{\sigma_{x} \sqrt{2}}\left(x-\frac{\lambda \sigma_{x}^{2}}{u}-u\left(t-t_{r}\right)\right)\right)-\right. \\
& \left.\operatorname{erf}\left(\frac{1}{\sigma_{x} \sqrt{2}}\left(x-\frac{\lambda \sigma_{x}^{2}}{u}-u t\right)\right)\right\}
\end{aligned}
$$

where

$$
\begin{gathered}
G=\frac{q}{\sqrt{8 \pi} u \sigma_{y} H} e^{-\frac{y^{2}}{2 \sigma_{y}^{2}}}\left[\vartheta_{3}\left(\frac{z+h}{2 H}, \frac{\sigma_{z}^{2}}{2 H^{2}}\right)+\vartheta_{3}\left(\frac{z-h}{2 H}, \frac{\sigma_{z}^{2}}{2 H^{2}}\right)\right] \\
e^{\frac{\lambda^{2} \sigma_{x}^{2}}{2 u^{2}}-\frac{\lambda x}{u}}
\end{gathered}
$$

and

$$
\begin{aligned}
& \vartheta_{3}(v, t)=\frac{1}{\sqrt{\pi t}} \sum_{k=-\infty}^{+\infty} e^{-\frac{(v+t)^{2}}{t}} \quad 0 \leq v \leq 1 \\
& \operatorname{erf}(z)=\frac{2}{\sqrt{\pi}} \int_{0}^{z} e^{-w^{2}} d w
\end{aligned}
$$

The dispersion coefficients $\sigma_{x}, \sigma_{y}, \sigma_{z}$ can be calculated according to different parameterisations [e.g. Gifford, 1976]. The PBL height H must be calculated considering convective and mechanical turbulence as explained for example in [Seibert et al., 2000]. The model calculates the effective source height as a consequence of the plume rise due to thermal buoyancy and mechanical moment [e.g. Briggs, 1972].

The input data required by the model concern the meteorology (wind speed, wind direction, stability class, PBL height, ...), the source (stack height, stack diameter, emission rate, emission temperature, ...), the domain (extension and number of grids), the output specification (how many maps, times for maps, receptor positions, ...).

TOXFLAM must be used over almost flat orography, with homogeneous meteorological and turbulent variables, and at distances up to about ten kilometres. 


\section{MAIN FEATURES OF THE MODELLING SYSTEM}

\subsection{Description}

The modelling system allows access to specific users authenticated by login and password. When a user logs in, the system reserves a specific disk portion for her/him, where the base map files, the output and the temporary files created during the simulations are stored.

Each user uploads the base maps of interest accessing to a specific section of the internet site. The base maps must have linear coordinates (special projections are not allowed), and are spatially referenced by the user by inserting the coordinates of the lower left and upper right corners. The scale of each base map is decided by the user as a function of the expected incident end point distance. Each user can upload all the domains of interest and carry out simulations over them. When at least one base map has been uploaded by the user, the simulation system can be used.

The simulation system contains a database with physical and chemical properties of the substances, and the information concerning the type and level of hazard of each substance (i.e. LFL, UFL, toxicity levels, ...). The variables included in the database are, for example, molar weight, pressure and temperature at critical point, coefficients for calculating the heat capacity of a vapour as function of the temperature, coefficients for calculating the vapour pressure as function of the temperature, heat of combustion, heat of vaporisation, toxicity levels, flammable levels and many other variables. These variables have been obtained from different references. When not directly available, some of them have been calculated using reliable relations indicated in the literature [e.g. Reid et al., 1987; Perry and Green, 1997]. The database currently contains about 50 substances and others will be added in the future. The database is currently used only for EXPSYS, for which the user selects the name of the substance involved in the accident and all its properties are automatically read (Figure 1). Simulations are currently limited to the substances in the database. TOXFLAM does not interact with the database, therefore the release of any substance can be simulated.

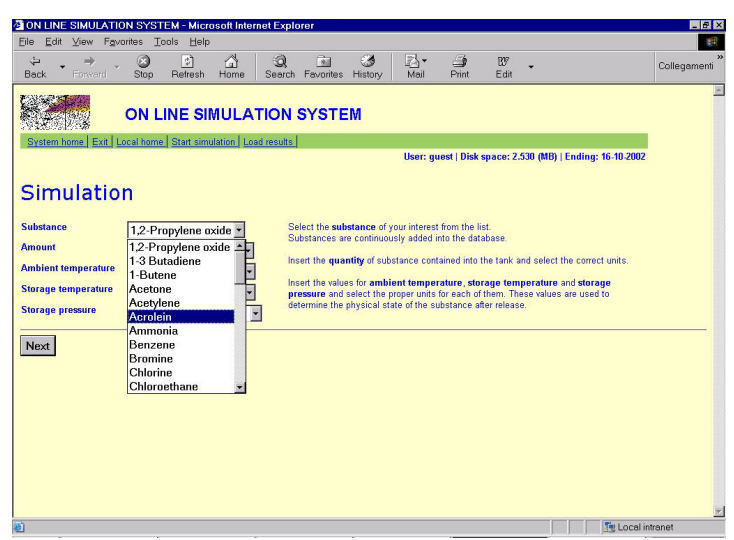

Figure 1. Example of input mask: selection of released substance for EXPSYS simulation.

The user interfaces of the modelling system have been designed to be as friendly as possible and to give all the necessary help to non-expert users, in particular in EXPSYS. For example the georeferentiation of the sources can be done by simply clicking over the base map (Figure 2), therefore the user is not required to know exactly the source coordinates.

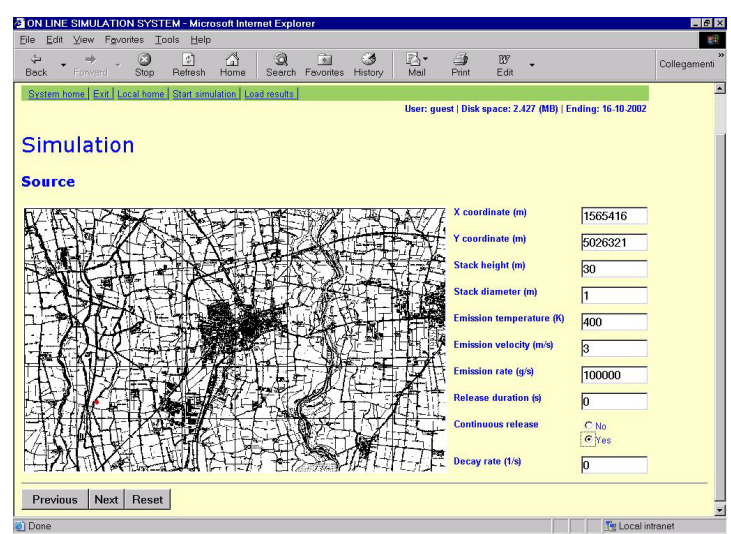

Figure 2. Example of input mask: definition of source parameters for TOXFLAM simulation.

The interface allows one to go forward and backward in carrying out a simulation, so that the user can modify input variables (substance released, quantity, release rate, meteorological conditions, ...). In this way it is possible to modify one variable at a time (the others are stored in memory by the system) and to see the effects on the output. As already mentioned, the system, when EXPSYS is used, gives as a result, for each type of accident, the area impacted by a specific effect (e.g. concentration above the toxicity level of the substance for a toxic release, thermal radiation above the limit to damage a specified target for a fire, etc.). This area is plotted over the map giving information useful to set up all the necessary on site and off site prevention measures or emergency plans. When TOXFLAM is used the results are the concentration levels superimposed 
on the base map (Figure 3). The TOXFLAM output file can also be downloaded and saved on the user's personal computer in order to be further analysed if necessary.

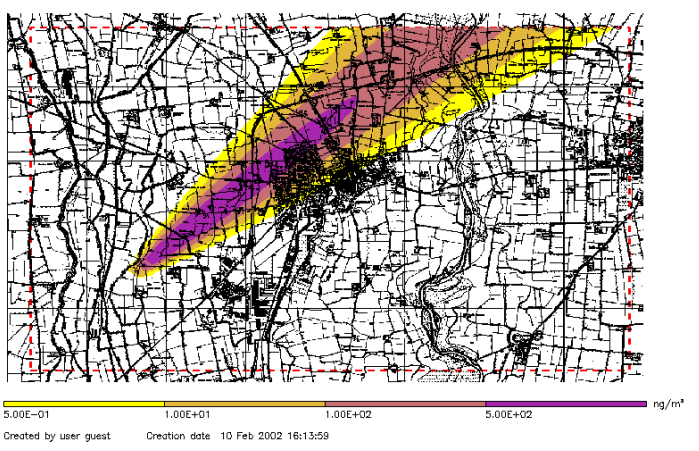

Figure 3. Example of graphical output from a TOXFLAM simulation.

All the simulation results can be saved in the user's personal folder. At any time they can be reloaded, deleted or used to set up another simulation with similar input data.

\subsection{Programming languages}

The modelling system is currently hosted under a personal computer (Server) with Linux operating system. All the user actions are managed by a series of Perl scripts capable for example of authenticating the users, sending email messages and modifying the user passwords. The most important purpose of the Perl scripts is to dynamically create the HTML pages, input masks of the models, as a function of the user actions or choices, and to launch the simulation models when all the input data are correctly specified. In order to avoid simulation errors due to incorrect input specifications, checks over the input values are carried out using the Javascript language. Javascript has been preferred to Perl for carrying out these checks because it acts directly on the user's machine (Client), avoiding superfluous work to the Server. Javascript is used for example to verify that some input are numeric, some other are not empty, the source coordinates are inside the specified domain, and other similar checks.

The two models, EXPSYS and TOXFLAM, are written in FORTRAN and compiled under Linux. The models could be written also in Perl, but FORTRAN has been preferred because it produces compiled programs, while Perl instructions are interpreted, therefore it guarantees more rapid executions. Moreover almost all the simulation models are written in FORTRAN, they have been used for long times and are, hopefully, error free. Therefore it is more convenient to use them as they are, avoiding recoding in other languages that requires time and could introduce potential programming errors.

The simple graphical representations of the EXPSYS output are obtained using the PerlMagick library, while the more complex graphical representation of the TOXFLAM results are obtained with Interactive Data Language (IDL(C) of Research Systems, Inc.

\subsection{Internet}

In the authors' viewpoint an internet based simulation system has some advantages with respect to single simulation models used off-line. First of all the simulation system is available on any platform, independently from the operating system, and it appears similar to any user, the only small differences are due to the different internet browsers (Netscape, MS Explorer, ...). Of course an internet connection must be available, but nowadays it is available almost everywhere, even in very rural sites without a cabled telephone line, if a portable computer and a GSM are available. This feature makes the system suitable to be used even on site, during a slowly evolving accident (e.g. the dispersion of a toxic cloud under low wind speed).

Moreover the graphical user interface created with HTML makes the models more friendly and easy to use. Other languages allow one to create graphical user interfaces (e.g. MS Visual Basic), but the wide diffusion of the internet and the familiarity of people with the most common browsers, should make the system of intuitive use. Another advantage is that all the users share a unique simulation tool, they can suggest modifications and improvements are readily available to all. There is no limit to the complexity of the models that can be installed on the Server, and the execution time depends only on the CPU speed and on the number of concurrent processes.

\section{FUTURE DEVELOPMENTS}

Currently EXPSYS treats the release of pure substances and it is not suitable for simulating releases of mixtures or compounds in solution. Since mixtures are often used in industries, one of the future developments will concern the release of non-pure compounds.

The internal database containing the chemical and physical properties of the substances currently considers about 50 chemical compounds. Other substances will be soon added since modern 
industry utilises hundreds of them. The database will be also enriched with information concerning the first aid to people injured by the release of a specific substance.

More complex models will be added to the system in the future. Of particular interest could be for example models allowing the treatment of multiple source releases, or models capable of simulating the dispersion of dense vapours. These models will probably require simulation times longer than those required by the models currently in the system. For this reason the user will launch the simulations as a background process and will be alerted, when they finish, by an email message or by an SMS message. At this time the user will connect again to the system and will download or simply see the simulation results.

\section{CONCLUSIONS}

An internet based simulation system has been described in this paper. The modelling tools currently contained in the system allow one to simulate the consequences of industrial accidents, and others will be added in the future. The system can be used almost everywhere provided and internet connection is available. It is completely platform independent and can be used on Unix, Linux, Windows or Macintosh operating systems. The modelling system can be used to set up emergency plans as consequence of different accidental scenarios. It gives both text and graphical (georeferenced) results in very short times, and allows one to go back and forward among the input masks keeping memory of all the input data. In this way the user will rapidly take into account input uncertainty modifying only some input data, maintaining the others unchanged, and redoing the simulation. The system, and its future evolutions, can be of great help to public authorities and private industries in setting up emergency plans in almost real time.

A similar simulation system could be tailored ad hoc for a specific industry, both to set up emergency plans or to be activated in emergency situations, and to monitor routine emissions from production plants. For this purpose the system can be improved with connections to a meteorological monitoring station and with monitors measuring smoke concentrations, flow rates and other stack parameters.

\section{Acknowledgments}

The authors wish to thank the Province of Milan (Italy) for its partial contribution to this work.

\section{References}

Bianconi R. and Tamponi M., A mathematical model of diffusion from a steady source of short duration in a finite mixing layer. Atmospheric Environment, 27A, 781-792, 1993.

Briggs G.A., Discussion of chimney plume in neutral and stable surroundings, Atmospheric Environment, 6, 507-510, 1972

Gifford F.A. Turbulent diffusion-Typing Schemes: A review, Nuclear Safety, 17, 68-86, 1976

Marshall V.C., Major chemical hazards. J. Wiley \& Sons., 1987.

Perry R.H. and Green D.W., Perry's chemical engineers' handbook. McGraw-Hill International Editions, 1997.

Quaranta N., De Martini A., Bellasio R., Bianconi R., Marioni M., A decision support system for the simulation of industrial accidents. Environmental Modelling \& Software, 2002, Accepted for publication.

Reid R.C., Prausnitz J.M. and Poling B.E., The properties of gases and liquids. $4^{\text {th }}$ ed. McGraw Hill, 1987.

Seibert, P., Beyrich, F., Gryning, S.-E., Joffre, S., Rasmussen, A., Tercier, P., Review and intercomparison of operational methods for the determination of the mixing height. Atmospheric Environment, 34(7), 1001-1027, 2000.

US-EPA, Risk management program guidance for offsite consequence analysis. EPA 550-B-99009, 1999.

Woodward J.L. and Mudan K.S., Liquid and gas discharge rates through holes in process vessels, Journal of Loss Prevention in Process Industry, 4, 161-165, 1991. 